\title{
Pentagon copying is more impaired in dementia with Lewy bodies than in Alzheimer's disease
}

\author{
T A Ala, L F Hughes, G A Kyrouac, M W Ghobrial, R J Elble
}

\begin{abstract}
Objectives-In many cases the clinical differentiation of patients with dementia with Lewy bodies (DLB) from those with Alzheimer's disease (AD) has been difficult. Because many neuropsychological studies have reported greater visuospatial/ constructional impairment in DLB than in AD, it was determined whether accuracy in copying the interlocking pentagons item on the mini mental state examination (MMSE) may be helpful in distinguishing patients with DLB from those with AD relatively early in the course of the dementia.

Methods-All cases of neuropathologically proved DLB and AD in the Center for Alzheimer Disease and Related Disorders brain bank were retrospectively reviewed, and the first available MMSE for each was retrieved. Only patients with MMSE scores $\geqslant 13$ were included, indicating mild to moderate dementia. The patients' copies of the interlocking pentagons were analyzed and graded as acceptable or unacceptable according to the original instructions for grading the MMSE.
\end{abstract}

Results-Seventeen patients with DLB and 27 patients with $A D$ were identified for whom MMSE with copies of the interlocking pentagons were available. Two patients with DLB (MMSEs 22 and 27) drew the pentagons acceptably, by contrast with 16 of the patients with $A D$ (MMSEs 13-28). An unacceptable copy was associated with DLB with a sensitivity of $88 \%$ and a specificity of $59 \%(p=0.002)$. Conclusions-For patients with MMSE scores $\geqslant 13$, an inability to accurately copy the pentagons suggests that the diagnosis is more likely DLB than AD. The results confirm the work of others on visuospatial/constructional impairment in DLB and indicate that this feature may be helpful in its diagnosis.

(F Neurol Neurosurg Psychiatry 2001;70:483-488)

Keywords: Alzheimer's disease; Lewy body disease; neuropsychological tests

In many cases the clinical differentiation of dementia with Lewy bodies (DLB) from Alzheimer's disease (AD) has been difficult. ${ }^{1-3}$ Whereas patients with the classic features of fluctuation, visual hallucinations, and parkinsonism may be readily recognised, many patients proved to have DLB at necropsy did not have these core symptoms and signs recognised during life.
There have been many studies of the neuropsychological profiles of patients with DLB, including some based on neuropathologically proved cases $^{4-8}$ and others based on clinically diagnosed cases. ${ }^{9-16}$ Despite these efforts, a reliable pattern to distinguish DLB from AD has not yet been identified. Many of these studies, however, have indicated that patients with DLB have greater visuospatial and constructional disorganisation than patients with AD. ${ }^{5} 689111214-16$ Deficits on tests of visuospatial ability are also mentioned in the consensus criteria for the clinical diagnosis of probable and possible DLB, ${ }^{17}$ but they have not been proposed as either a supportive or core feature.

Considering this prominent visuospatial/ constructional impairment in DLB and the current difficulty in its clinical diagnosis, we investigated our patients with DLB and AD to see if this characteristic may have helped to differentiate the two diseases. We also questioned if it may have been detected relatively early in the course of the dementia. Because many of our cases included clinical records with a mini mental state examination ${ }^{18}$ (MMSE), and as the MMSE includes a visuospatial/ constructional test in its interlocking pentagon copying item, we determined if the MMSE may have provided an early clue to differentiate DLB from AD. To avoid the problems inherent in the clinical diagnosis of DLB and AD, we studied only cases that had been neuropathologically proved, having come to necropsy through our brain bank programme.

\section{Methods}

All cases with the neuropathological diagnoses of DLB and AD that had been received in the Center for Alzheimer Disease and Related Disorders Brain Bank since its inception in 1987 were reviewed in this retrospective study. Consent for necropsy and research usage of clinical records was obtained from the families of the patients in accordance with the guidelines of the Southern Illinois University School of Medicine Springfield Committee for Research Involving Human Subjects.

The neuropathological diagnosis of DLB was based on the presence of cortical Lewy bodies (LBs), identified by either haematoxylin and eosin or antiubiquitin stains. The relative number or distribution of cortical LBs or the presence of concomitant Alzheimer's changes were not factors in study inclusion. The neuropathological diagnosis of $\mathrm{AD}$ was based on the presence of both neuritic plaques and neurofibrillary tangles in the neocortex, consistent with current guidelines. ${ }^{19}$ The cases were also reviewed to exclude those that had 
Table 1 Criteria for grading the interlocking pentagons on the mini mental state examination $^{18}$

Original criteria ${ }^{18}$ :

To be acceptable, all 10 angles must be present and two must intersect

Tremor and rotation are ignored

Relaxed criteria:

To be acceptable, there must be two figures that seem to be intersecting. At least one of the figures must have five angles

Tremor, rotation, relative size, and symmetry are ignored

Consider only lines that the patient has drawn (ignore any lines from the original model that the patient may have included as part of the copy)

If the patient has drawn more than one copy, grade the best copy.

Table 2 Patient demographics

\begin{tabular}{llll}
\hline Characteristic & Lewy body group & Alzheimer's group & p Value \\
\hline No of patients & 17 & 27 & \\
Male/female & $11 / 6$ & $13 / 14$ & 0.36 \\
Age (mean (SD)) & $75.0(7.2)$ & $79.7(7.6)$ & 0.047 \\
Years of education (mean (SD)) & $13.6(3.3)$ & $12.1(3.3)$ & 0.14 \\
MMSE score (mean (SD)) & $21.8(4.5)$ & $20.9(4.3)$ & 0.54 \\
\hline
\end{tabular}

MMSE $=$ Mini mental state examination. ${ }^{18}$

infarcts or other neuropathology that may have had clinical significance.

All MMSE forms that were found in the clinical records of the cases were reviewed. We considered only complete MMSE forms that documented the patient's responses to each question, including the patient's attempt(s) to copy the interlocking pentagons. In this study we included only those patients who scored $\geqslant 13$ on their first available MMSE, indicating mild to moderate dementia. Later MMSE scores or attempts at copying the pentagons that may have been better were not considered. If there was more than one pentagon copying attempt on the MMSE form, the most accurate copy was used.

Each patient's copy of the pentagons was graded according to two different criteria. The first criteria, or "original" grading criteria, are presented in table 1 and are identical to those first published with the MMSE. ${ }^{18}$ All 10 angles must be present and two must intersect to be acceptable. A second set of criteria was also studied to see if either sensitivity or specificity could be improved. This second set, or "relaxed" grading criteria, allows for some inaccuracy in either the number of angles or the intersection. The relaxed criteria are also presented in table 1. Applying either criteria results in an acceptable or an unacceptable copy.

To eliminate any possible bias introduced by our knowledge of the patients' diagnoses and overall MMSE scores, a group of five experienced psychometricians independently graded the patients' copies of the pentagons. The copies were presented randomly without indication of diagnosis or MMSE score. A majority vote of the five psychometricians determined whether or not a given copy was acceptable or unacceptable.

\section{STATISTICAL ANALYSIS}

Mean differences in age, years of education, and overall MMSE scores between the two patient groups were analyzed using analysis of variance (ANOVA). The patients' overall scores on the MMSEs were used in the analysis, with no adjustment made for the point that the patient may have scored on the pentagon copying item. The determination of association between dichotomous variables such as sex, diagnosis, and pentagon grade (acceptable, unacceptable) was made with Fisher's exact test. Significance was defined at the $\mathrm{p}=0.05$ level. Reliability among the psychometricians was assessed with Pearson correlations and Cronbach's $\alpha$.

Sensitivity was calculated by dividing the number of patients with DLB who had an unacceptable copy by the total number of patients with DLB. Specificity was calculated by dividing the number of patients with $\mathrm{AD}$

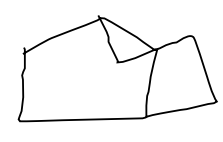

LB-1 (29--)

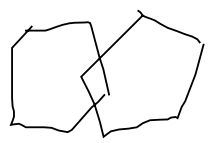

LB-2 $(29-+)$

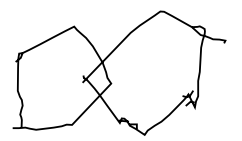

LB-3 $(27++)$

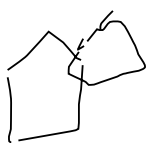

LB-4 $(27-+)$

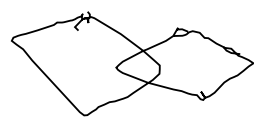

LB-5 (23--)

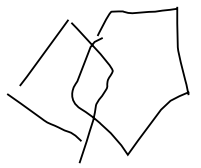

LB-6 $(23-+)$

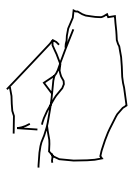

LB-12 $(20-+)$

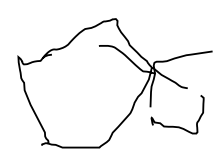

LB-7 (22--)

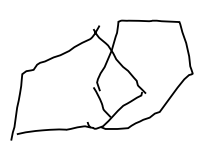

LB-13 $(20-+)$

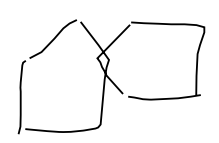

LB-8 $(22++)$

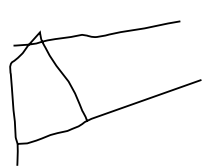

LB-14 (19--)

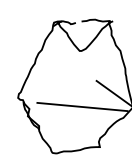

LB-9 (22 - -)

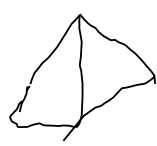

LB-15 (18 - -)

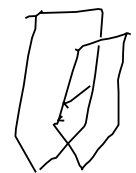

LB-10 (21 - +)
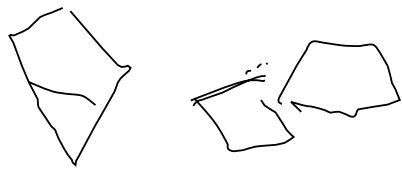

LB-16 (14 - -)

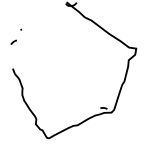

LB-11 (21 - -)

LB-17 (13 - -)

Figure 1 Copies of the interlocking pentagons performed by the patients in the DLB group. The code beneath each copy indicates the patient identifier, the patient's overall score on the mini mental state examination (MMSE) ${ }^{18}$, and how the copy was graded according to the original grading criteria (first grade) and the relaxed grading criteria (second grade). For example, "LB-2 (29- +)" indicates that the copy was done by patient 2 in the LBD group, that the patient's overall MMSE score was 29, and that the copy was considered unacceptable (-) by the original criteria and acceptable (+) by the relaxed criteria. See table 1 for details of the two grading criteria. The models shown to the patients for them to copy are not included. The original copies have been reduced in size for this figure. 


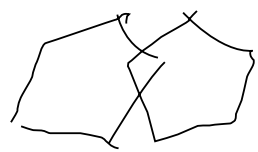

AD-1 $(28++)$

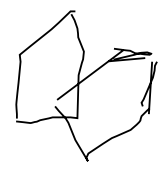

AD-2 $(28++)$

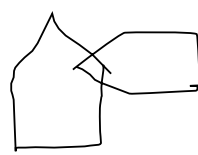

AD-3 $(28++)$

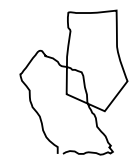

AD-4 $(26++)$

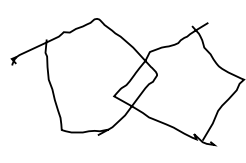

AD-5 $(26++)$

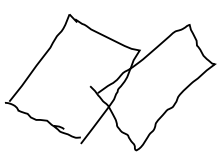

AD-6 (24--)
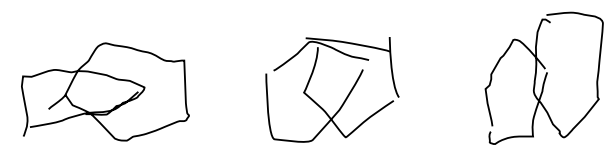

AD-7 $(24-+)$

AD-8 $(24++)$

AD-9 $(23++)$
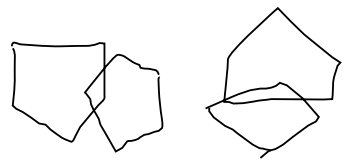

AD-10 $(23++)$

AD-11 $(22-+)$
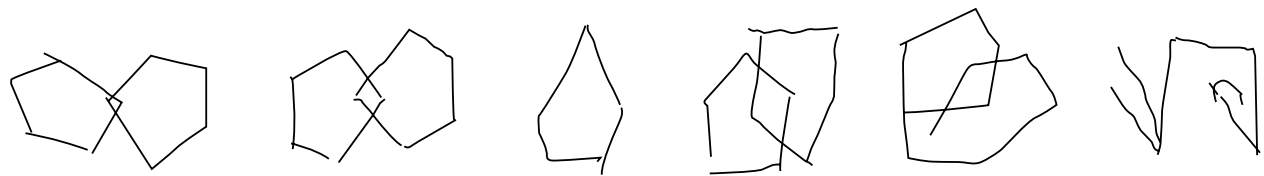

AD-12 $(22++)$

AD-13 $(21++$

AD-14 (20 - -)

AD-15 $(20++)$

AD-16 (20 - -)

AD-17 (20 - - )

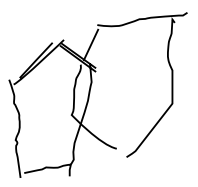

AD-18 $(20++)$

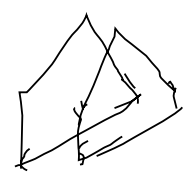

AD-23 (16 - +)

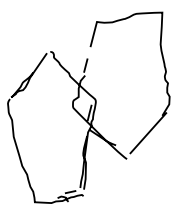

AD-19 (19++)

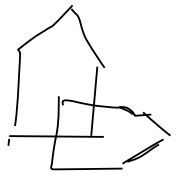

AD-24 $(15++)$

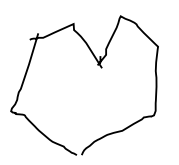

AD-20 (19 - -)

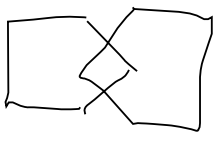

AD-21 $(18++)$

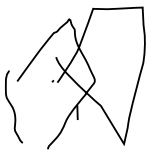

AD-22 $(18-+)$

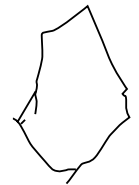

AD-25 (15 - -)

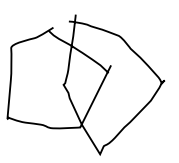

AD-26 $(13++)$

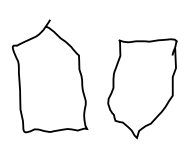

AD-27 (13--)

Figure 2 Copies of the interlocking pentagons performed by the patients in the AD group. See the legend for fig 1 for an explanation of the code beneath each copy. The models shown to the patients for them to copy are not included. The original copies have been reduced in size for this figure.

who had an acceptable copy by the total number of patients with AD. A commercial statistical program was used to calculate $95 \%$ confidence intervals (95\% CIs) (GraphPad InStat version 3.01 for Windows 95, GraphPad Software, San Diego, CA, USA).

To control for confounding factors such as differences in MMSE score, age, or education between the two groups or differences between the psychometrists, a third set of grading criteria was devised by which the psychometrists gave each pentagon copy a score from 0 to 3 points. Three points indicated that a copy met the original grading method criteria, and two points indicated that a copy met the relaxed criteria. One point was given if the copy seemed to have two intersecting figures, and zero points were given for less accurate copies. An analysis of covariance (ANCOVA), with MMSE score, age, and education as covariants, was used to compare the copy scores of the two patient groups.

\section{Results}

We identified 17 cases of DLB and 27 of AD for which we had MMSEs with copies of the interlocking pentagons. The demographic characteristics of the patients are presented in table 2. There were no significant differences between the two groups for sex, years of education, or overall scores on the MMSE. The mean age of the patients with DLB was about 5 years younger than that of the patients with AD (75.0 79.7 years, $\mathrm{p}=0.047$ ). Reproductions of the DLB group's pentagon copies are presented according to overall MMSE score in figure 1 and those of the $\mathrm{AD}$ group in figure 2 .

Using the original criteria for grading the pentagons, two of the DLB group (LB-3 and LB-8, MMSEs 27 and 22, fig 1) had acceptable copies, by contrast with 16 of the AD group (MMSEs 13-28). A graph showing each patient's copy grade and his or her MMSE score is presented in figure 3 . The results were significant (Fisher's exact test, $\mathrm{p}=0.002$ ), with an unacceptable copy of the pentagons being associated with DLB with a sensitivity of $88 \%$ (95\% CI $0.64-0.99)$ and a specificity of $59 \%$ (95\% CI 0.39-0.78).

Using the relaxed criteria, eight of the DLB group (MMSEs 20-29) had acceptable copies by contrast with 20 of the AD group (MMSEs 


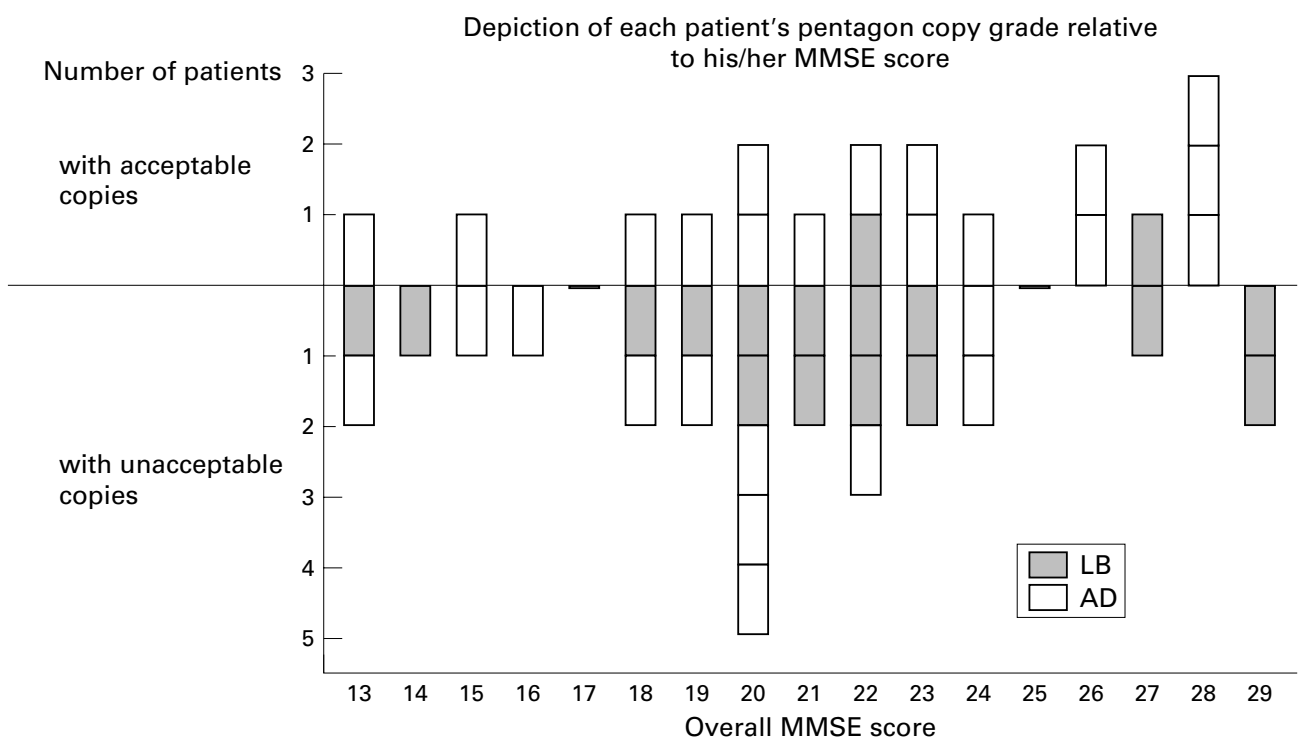

Figure 3 Each patient's grade on his or her pentagon copy relative to his or her overall score on the mini mental state examination (MMSE). ${ }^{18}$ The original grading criteria were used (see table 1 for description). LB=Patients with dementia with Lewy bodies; $A D=$ patients with Alzheimer's disease.

13-28). Although the results were not significant (Fisher's exact test, $p=0.11$ ), by these criteria an unacceptable copy was associated with DLB with a sensitivity of $53 \%$ and a specificity of $74 \%$.

Using the 0-3 grading system and controlling for age, years of education, and MMSE score, ANCOVA confirmed that the DLB group copied the pentagons significantly less accurately than the $\mathrm{AD}$ group $(\mathrm{p}=0.003)$. As in this instance each of the three covariates were biased towards favouring the DLB group (younger ages, more education, and higher MMSE scores), the analysis indicated an even greater adjusted mean difference between the scores of the two groups than when the variables were left uncontrolled. The analysis showed no statistical differences between the scores of the five psychometricians. Intercorrelations between psychometricians ranged from 0.88 to 0.95 . Cronbach's $\alpha$ was 0.98 .

At the time of the MMSE the clinical diagnoses of 13 of the 17 patients in the DLB group and of all 27 of the patients in the $\mathrm{AD}$ group were memory disorder, possible AD, or probable AD. Two patients with DLB were considered to have both Parkinson's disease (PD) and AD (LB-3 and LB-17, fig 1), and two were considered as having PD (LB-2 and LB-4, fig 1). In each of these four cases the onset of the patient's cognitive impairment preceded the symptoms of parkinsonism by at least 1 year. The year of administration of the MMSEs ranged from 1987 to 1997 for the DLB group (median 1991) and from 1987 to 1998 for the AD group (median 1991).

One case listed in our brain bank as AD was excluded because histopathological slides were no longer available for review to see if there may have been cortical LBs present. The neuropathology report for this 1987 case indicated the diagnosis as $\mathrm{AD}$ with substantia nigra LBs; the patient's MMSE score was 16, with an unacceptable pentagon copy by the original method and an acceptable copy by the relaxed method. Three of the AD group had marked degeneration of the substantia nigra without LBs (AD-2, AD-5, and AD-21, fig 2). Two patients with DLB were excluded because the clinical records indicated that the patients had PD with dementia, defined as those who had onset of parkinsonism at least 1 year before onset of the dementia. ${ }^{17}$ No cases were excluded due to infarcts. None of the additional cases of $\mathrm{AD}$ and DLB in our brain bank with initial MMSE scores less than 13 had acceptable pentagon copies.

The MMSEs were administered by many different examiners. Seven different printed models of the interlocking pentagons were used in this study, and in 19 patients the examiners drew the model at the time of the MMSE administration. No adjustment was made for possible arthritic, orthopaedic, visual, or other physical impairments of the patients. For 13 patients the examiners allowed multiple trials. In only one instance did we change an MMSE score, when we reduced a score of 30 to 29 because it was clear that the patient's copy did not fulfil the original grading criteria (LB-2, fig $1)$.

\section{Discussion}

Our results are consistent with the findings of many other studies that have compared the neuropsychological profiles of DLB and patients with $\mathrm{AD}$; patients with DLB have greater visuospatial/constructional impairment than patients with AD. Furthermore, as others have also found, ${ }^{4} 5814$ in many cases this impairment may be demonstrated relatively early in the course of the dementia.

Although not the primary focus of our study, our results also suggest that the pentagon copying item of the MMSE may be useful in a diagnostic sense. With the caveat that a given patient's performance on a single task should not be considered definitive, a patient who has a relatively high MMSE score and prominent 
difficulty in copying the interlocking pentagons has a greater likelihood of having DLB than AD. For example, three of the four patients with DLB with MMSE scores greater than 25 had unacceptable copies by contrast with none of the five patients with AD. Conversely, a patient who copies the pentagons well but has a relatively low MMSE score has a greater likelihood of having AD than DLB.

Even though the results of the relaxed grading criteria were not significant $(p=0.11)$, they are helpful in stressing that the grading of a patient's pentagon copy should be strict for it to be most meaningful in identifying potential patients with DLB. An unacceptable copy by the relaxed criteria detected only $53 \%$ of the patients with DLB (sensitivity) by contrast with $88 \%$ by the original criteria.

That this visuospatial/constructional impairment of DLB has been identified in necropsy proved cases irrespective of any other clinical details is very interesting, for it suggests that it may be a characteristic that is independent of the current core DLB clinical features of fluctuation, visual hallucinations, and parkinsonism. ${ }^{17}$ If proved in future studies, including visuospatial/constructional impairment as an additional core criterion could improve the sensitivity of the current criteria to detect additional patients. The sensitivity of the current criteria is a weakness in its usefulness. ${ }^{132021}$ The potential importance of this feature is further emphasised by the necropsy proved studies that have recognised it even in patients with DLB clinically diagnosed as having $\mathrm{AD} .{ }^{6} 8$

One limitation of the current study is our lack of control or even documentation of how the MMSEs were administered. We do not think that this is a major weakness, nevertheless, as it could be argued that our results would reflect those more likely obtained in a non-academic clinical setting. Future study should include a standardised model and administration procedure. We also caution that we only studied DLB and patients with AD. We did not include normal controls, nor did we study patients with dementia caused by frontotemporal dementia, vascular dementia, mixed dementias, or other dementing illnesses.

Although most of the other studies of visuospatial/constructional abilities have shown greater impairment in DLB than in AD, two ${ }^{412}$ have included MMSE pentagon copying analyses that did not show a difference. The first, ${ }^{4}$ a study of necropsy proved patients, found no significant difference on individual MMSE component performance when 13 patients with DLB were compared with 13 patients with AD. Pentagon copying data and mean MMSE scores were not reported. This study also did not identify differences between the DLB and AD groups using other constructional measures, yet when subgroups with mild to moderate dementia were examined, the difference became significant at $p<0.02$. The second, ${ }^{12}$ a study of clinically diagnosed patients, reported that the performance of 16 patients with DLB on this MMSE item did not differ statistically from that of 25 patients with AD. No pentagon copying data were presented, and the mean overall MMSE scores were substantially lower than ours: 12.5 for DLB and 13.4 for AD.

One relatively large necropsy proved study ${ }^{7}$ that did not find significant differences in clock drawing and constructional praxis included 24 cases of DLB and 71 of AD, all of which had been clinically diagnosed as $\mathrm{AD}$. By contrast with our results, the data from these patients were obtained at their last clinical visit rather their first, and their median MMSE scores were much lower: 9.0 for DLB and 10.5 for $\mathrm{AD}$.

One additional study ${ }^{22}$ reporting negative visuospatial/constructional results concluded that clock face drawing was unlikely to be useful in differentiating DLB and AD. We argue that this study, which included only clinically diagnosed patients, may unknowingly have included patients with DLB within its AD group, as 77 patients with $\mathrm{AD}$ were compared with one patient with DLB.

Our findings raise interesting issues that could be investigated in future studies. For example, we did not attempt to consider a mechanism for this difference between DLB and $\mathrm{AD}$. A quantitative study of the neuropathology of our cases might detect occipital changes that reflect the occipital hypometabolism that other investigators have noted on $\mathrm{PET}^{23}$ and SPECT ${ }^{24}$ imaging tests. There also may be other constructional tests that are more discriminating than the interlocking pentagons.

Although very difficult in a retrospective study, another investigation into our patient records could focus on other potential clinical correlates, such as whether the patients with DLB had a greater tendency to become lost or had more difficulty with other visuospatial or constructional tasks. It would also be worthwhile to see if our current pentagon copying data may correlate with either the data from other constructional tests such as clock drawing or with other non-constructional neuropsychological data that we may have. Perhaps the most important next research step will be to determine if this feature is indeed independent of the other well known characteristics of $\mathrm{DLB}$, in which case adding this as a feature to the clinical criteria for DLB may improve the sensitivity of the guidelines.

We are indebted to the neuropsychology section of the SIU Center for Alzheimer Disease and Related Disorders for providing their services for the grading of the pentagon copies. providing their services for the grading of the pentagon copies.
Fourteen of the patients in this study were assessed by Fourteen of the patients in this study were assessed by
coordinators in our Primary Provider Network: Robin Winnell from Bloomington-Normal; Brenda Sayre from Macomb; and Linda Fehr, and Julie O'Brien from Peoria. We also thank Frances Schaefer and Sandy Vicari of the Research Division of the SIU Department of Psychiatry for providing clinical records for two of the patients. This study was supported in part by a gift from the family of Harriss Malan and by the Illinois Department of Public Health's Regional Alzheimer Disease Assistance Center programme.

1 McKeith IG, O'Brien JT, Ballard C. Diagnosing dementia with Lewy bodies. Lancet 1999;354:1227-8.

2 Papka M, Rubio A, Schiffer RB, et al. Lewy body disease: can we diagnose it? $f$ Neuropsychiatry Clin Neurosci 1998;10:405-12.

3 Weiner MF. Dementia associated with Lewy bodies. dilemmas and directions. Arch Neurol 1999;56:1441-2.

4 Connor DJ, Salmon DP, Sandy TJ, et al. Cognitive profiles of autopsy-confirmed Lewy body variant vs pure Alzheimer disease. Arch Neurol 1998;55:994-1000. 
5 Galasko D, Katzman R, Salmon DP, et al. Clinical and neuropathological findings in Lewy body dementia. Brain Cogn ropathological fin

6 Hansen L, Salmon D, Galasko D, et al. The Lewy body variant of Alzheimer's disease: a clinical and pathologic entity. Neurology 1990;40:1-8.

7 Heyman A, Fillenbaum GG, Gearing M, et al. Comparison of Lewy body variant of Alzheimer's disease with pure Alzheimer's disease: Consortium to Establish a Registry for Alzheimer's Disease, Part XIX. Neurology 1999;52:1839-44.

8 Salmon DP, Galasko D, Hansen LA, et al. Neuropsychological deficits associated with diffuse Lewy body disease. Brain Cogn 1996;31:148-65.

9 Ballard CG, Ayre G, O’Brien J, et al. Simple standardised neuropsychological assessments aid in the differential diagnosis of dementia with Lewy bodies from Alzheimer's disosis and vascular dementia. Dement Geriatr Cogn Disord 1999:10:104-8

10 Galloway PH, Sahgal A, McKeith IG, et al. Visual pattern recognition memory and learning deficits in senile dementias recognition memory and learning deficits in senile dementias

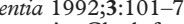

11 Gnanalingham KK, Byrne EJ, Thornton A. Clock-face drawing to differentiate Lewy body and Alzheimer type

12 Gnanalingham KK, Byrne EJ, Thornton A, et al. Motor and cognitive function in Lewy body dementia: comparison with Alzheimer's and Parkinson's diseases. 7 Neurol Neurosurg Psychiatry 1997;62:243-52.

13 Graham C, Ballard C, Saad K. Variables which distinguish patients fulfilling clinical criteria for dementia with Lewy bodies from those with Alzheimer's disease. Int $\mathcal{f}$ Geriat Psychiatry 1997;12:314-18.

14 Mori E, Shimomura T, Fujimori M, et al. Visuoperceptual impairment in dementia with Lewy bodies. Arch Neurol 2000;57:489-93.
15 Shimomura T, Mori E, Yamashita $\mathrm{H}$, et al. Cognitive loss in dementia with Lewy bodies and Alzheimer disease. Arch Neurol 1998;55:1547-52.

16 Walker Z, Allen RL, Shergill S, et al. Neuropsychological performance in Lewy body dementia and Alzheimer's disease. Br F Psychiatry 1997;170:156-8.

17 McKeith IG, Galasko D, Kosaka K, et al. Consensus guidelines for the clinical and pathological diagnosis of dementia with Lewy bodies (DLB): report of the Consortium on DLB International Workshop. Neurology 1996;47:111324.

18 Folstein MF, Folstein SE, McHugh PR. Mini-mental state. A practical method for grading the cognitive state of patients for the clinician. $\mathcal{F}$ Psychol Res 1975;12:189-98.

19 Consensus recommendations for the postmortem diagnosis of Alzheimer's disease. Neurobiol Aging 1997;18:S1-2.

20 Luis CA, Barker WW, Gajaraj K, et al. Sensitivity and specificity of three clinical criteria for dementia with Lewy bodies in an autopsy-verified sample. Int F Geriatr Psychiatry 1999;14:526-33.

21 Verghese J, Crystal HA, Dickson DW, et al. Validity of clinical criteria for the diagnosis of dementia with Lewy bodies. Neurology 1999;53:1974-82.

22 Swanwick GRI, Coen RF, Maguire CP, et al. Clock-face drawing to differentiate dementia syndrome. Lancet 1996; 347:1115.

23 Imamura T, Ishii K, Sasaki M, et al. Regional cerebral glucose metabolism in dementia with Lewy bodies and Alzheimer's disease: a comparative study using positron emission tomography. Neurosci Lett 1997;235:49-52.

24 Ishii K, Yamaji S, Kitagaki H, et al. Regional cerebral blood flow difference between dementia with Lewy bodies and AD. Neurology 1999;53:413-6.

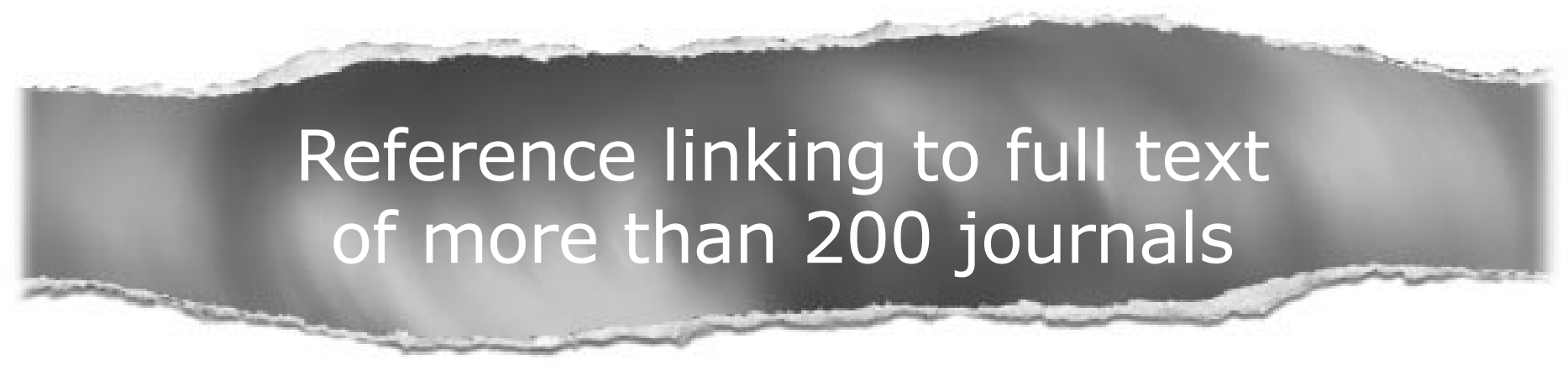

Toll free links

You can access the FULL TEXT of articles cited in the Journal of Neurology, Neurosurgery, and Psychiatry online if the citation is to one of the more than 200 journals hosted by HighWire (http://highwire.stanford.edu) without a subscription to that journal.

There are also direct links from references to the Medline abstract for other titles.

www.jnnp.com 